\title{
AUTHOR INDEX \\ Volume 4, 1994
}

Abraham, R., see Gardini

Afraimovich, V.S. \& Nekorkin, V.I., Chaos of travelling waves in a discrete chain of diffusively coupled maps

Aguirre, L.A. \& Billings, S.A., Validating identified nonlinear models with chaotic dynamics

Aguirre, L.A. \& Billings, S.A., Discrete reconstruction of strange attractors of Chua's circuit

Aguirre, L.A., Some remarks on structure selection for nonlinear models

Aihara, K., see Sakai

Alonso, M., see Pêrez-Muñuzuri

Amritkar, R.E., see John

Anishchenko, V.S., Neiman, A.B. \& Chua, L.O., Chaos-Chaos intermittency and $1 / f$ noise in Chua's circuit

Anishchenko, V.S., Safonova, M.A. \& Chua, L.O., Stochastic resonance in Chua's circuit driven by amplitude or frequency modulated signals

Anishchenko, V.S., Safonova, M.A., Feudel, U. \& Kurths, J., Bifurcations and transition to chaos through three-dimensional tori

Arecchi, F.T., Basti, G., Boccaletti, S. \& Perrone, A.L., Adaptive recognition of chaos

Arecchi, F.T., Boccaletti, S., Giacomelli, G., Puccione, G.P., Ramazza, P.L. \& Residori, S., Boundary dominated versus bulk dominated regime in optical space-time complexity

Arneodo, A., see Muzy

Atela, P. \& McLachlan, R.I., Global behavior of the charged isosceles three-body problem

Babu Joseph, K., see Valsamma

Bacry, E., see Muzy

Basti, G., see Arecchi

Bedding, S., A new strange attractor in three dimensions

Beige, H., see Brauer

Bernhardt, P.A., Communications using chaotic frequency modulation

Bestehorn, M., Pattern selection in Benard-Marangoni convection

Biktashev, V., Krinsky, V. \& Haken, H., A wave approach to pattern recognition (with application to optical character recognition)

Billings, S.A., see Aguirre

Billings, S.A., see Aguirre

Bishop, S.R., see Clifford

Blochwitz, S., see Brauer

Boccaletti, S., see Arecchi

Boccaletti, S., see Arecchi

Bonhoeffer, S., see Nowak

Brauer, E., Blochwitz, S. \& Beige, H., Periodic windows inside chaos - experiment versus theory

Braun, T., Lisboa, J.A., Characterization of homoclinic chaos in a glow discharge through return maps

Breeden, J.L. \& Packard, N.H., A learning algorithm for optimal representation of experimental data

Burguete, J., see Maza-Ozcoidi

Careta, A., Sancho, J.M. \& Sagues, F., Dynamics of reactiondiffusion interfaces under stochastic convection: Preliminary results

Castro, S.B.S.D., Mode interactions with Spherical symmetry

Cathala, J.C., see Mira

Celka, P., Experimental verification of Pyragas's chaos control method applied to Chua's circuit
Chaitin, G.J., Randomness and complexity in pure mathematics

Champneys, A.R. \& Kuznetsov, Yu A., Numerical detection and continuation of codimension-two homoclinic bifurcations

Champneys, A.R., Subsidiary homoclinic orbits to a saddle-focus for reversible systems

Chen, G., Optimal control of chaotic systems

Chern, J.-L., see Otsuka

Chialina, S., Hasler, M. \& Premoli, A., Fast and accurate calculation of Lyapunov exponents for piecewise-linear systems

Christov, C.I. \& Velarde, M.G., Inelastic interaction of Boussinesq solitons

Chu, P.H., Milton, J.G. \& Cowan, J.D., Connectivity and the dynamics of integrate-and-fire neural networks

Chua, L.O., see Anishchenko

Chua, L.O., see Anishchenko

Chua, L.O., see Kapitaniak

Chua, L.O., see Kapitaniak

Chua, L.O., see Kocarev

Chua, L.O., see Zheleznyak

Chua, L.O., see Itoh

Chua, L.O., see $W u$

Chua, L.O., see Murali

Chua, L.O., see Pérez-Muñuzuri

Chua, L.O., see Itoh

Chua, L.O., see Pivka

Clifford, M.J. \& Bishop, S.R., Bifurcational precedences for parametric escape from a symmetric potential well

Coullet, P. \& Plaza, F., Excitable spiral waves in nematic liquid crystals

Cowan, J.D., see Chu

Crowley, M.F., see Petrov

Cuomo, K.M., Synthesizing self-synchronizing chaotic arrays

D'Angelo, E.J., see Jeffery

de Angeli, A., see Tesi

de Kepper, P., Perraud, J.J., Rudovics, B. \& Dulos, E., Experimental study of stationary Turing patterns and of their interaction with travelling waves in a chemical system

de Sousa Vieira, M., Lichtenberg, A.J. \& Lieberman, M.A., Nonlinear dynamics of digital phase-locked loops with delay

de Sousa Vieira, M., Lichtenberg, A.J. \& Lieberman, M.A., Selfsynchronization of many coupled oscillators

Dedieu, H. \& Ogorzalek, M., Controlling chaos in Chua's circuit via sampled inputs

Del Río, E., Velarde, M.G., Rodríguez-Lozano, A., Rul'kov, N.F. \& Volkovskii, A.R., Experimental evidence for synchronous chaotic behavior of nonlinear oscillators with unidirectional or mutual chaotic driving

Deng, B., Constructing homoclinic orbits and chaotic attractors

Dialetis, D., see Pavlos

Diercksen, G.H.F., see von Milczewski

Dimovski, D., see Kocarev

Ding, J. \& Tam, S.-W., Asymptotic power spectrum analysis of chaotic behavior in fluidized beds

Ding, M. \& Kelso, J.A.S., Phase-resetting map and the dynamics of quasi-periodically forced biological oscillators

Doerner, R., see Heng

Doerner, R., Hübinger, B., Heng, H. \& Martienssen, W., Approaching nonlinear dynamics by studying the motion of a pendulum. II. Analyzing chaotic motion 
Doerner, R., see Hübinger

Dolinchuk, S.G., Zadorozhnii, V.I. \& Fedorchenko, A.M., Hierarchy of instabilities and solitons in an acoustic distributed system with feedback

Dowell, E.H., see Katz

Dullin, H.R., Juhnke, M. \& Richter, P.H., Action integrals and energy surfaces of the Kovalevskaya top

Dulos, E., see De Kepper

Eleonsky, V.M., see Popkov

Englisch, V. \& Lauterborn, W., The winding-number limit of period-doubling cascades derived as Farey-fraction

Fedorchenko, A.M., see Dolinchuk

Fernández-García, G., see Gómez-Gesteira

Fernández-Vela, J. \& Pérez-García, C., Oscillatory instabilities in binary mixtures for positive separation ratio

Ferre, F., see Sornette

Feudel, U., see Anishchenko

Fournier-Prunaret, D., see Gardini

Fournier-Prunaret, D., see Mira

Frankel, M.L. \& Roytburd, V., Dynamical portrait of a model of thermal instability: cascades, chaos, reversed cascades and infinite period bifurcations

Friedrich, R., see Neufeld

García-Ojalvo, J. \& Sancho, J.M., Effects of multiplicative noise on a stochastic Swift-Hohenberg equation

Garcimartin, A., see Mindlin

Gardini, L., Abraham, R., Record, R.J. \& Fournier-Prunaret, D., $A$ double logistic map

Gardini, L., see Mira

Gatermann, K. \& Werner, B., Group theoretical mode interactions with different symmetries

Gaveau, B. \& Moreau, M., Resonance effects for chemical reactivity in complex media

Genesio, R., see Tesi

Giacomelli, G., see Arecchi

Glass, L. \& Zeng, W., Bifurcations in flat-topped maps and the control of cardiac chaos

Gómez-Gesteira, M., see Hanusse

Gómez-Gesteira, M., Fernández-García, G., Muñuzuri, A.P., PérezMuñuzuri, V., Krinsky, V.I., Starmer, C.F. \& Pérez-Villar, V., Spiral formation in a Belousov-Zhabotinsky medium by premature re-excitation: Vulnerability

Gómez-Gesteira, M., see Muñuzuri

González-Viñas, J. \& Salán, J., Resonantly forced surface waves in a natterer tube. Patterns, defects and squeezes

Grigoryan, Yu I., see Papanyan

Grimson, M.J., Spatiotemporal growth of bacterial colonies

Grohs, J., see Zhang

Guerra, J.M., see Pérez-García

Gupta, S.D., see Pande

Haken, H., see Biktashev

Haken, H., Synergetics: From pattern formation to pattern analysis and pattern recognition

Hanusse, P., Pérez-Muñuzuri, V. \& Gómez-Gesteira, M., Relaxation behavior and pattern formation in reactiondiffusion systems

Hasler, M., see Chialina

Hastings, S.P., A "shooting" approach to chaos

Heng, H., Doerner, R., Hübinger, B. \& Martienssen, W. Approaching nonlinear dynamics by studying the motion of a pendulum. 1. Observing trajectories in state space

Heng, H., see Doerner

Heng, H., see Hübinger

Herrero, H. \& Riecke, H., Front structures in a real GinzburgLandau equation coupled to a mean field
Holzfuss, J., see Parlitz

1715

Huibinger, B., see Heng

Hübinger, B., see Doerner

Hübinger, B., Doerner, R., Heng, H. \& Martienssen, W., Approaching nonlinear dynamics by studying the motion of a pendulum. III. Predictability and control of chaotic motion

Ishiyama, F. \& Otsuka, K., Intensity probability distribution of spiking-mode oscillations in lasers: power-law universality

Issler, H., see Zhang

Itoh, M., Murakami, H. \& Chua, L.O., Experimental control of chaos in Chua's circuit via tunnels

Itoh, M. \& Murakami, H., Chaos and canards in the van der Pol equation with periodic forcing

Itoh, M., Murakami, H. \& Chua, L.O., Experimental study of forced Chua's oscillator

Iwanaga, H., see Sumida

Jaeger, N.I., see Otterstedt

Jeffery, M. \& D'Angelo, E.J., Optical vortex interaction in lasers

John, J.K. \& Amritkar, R.E., Synchronization by feedback and adaptive control

Johnson, S.D., Simple hybrid systems

Juhnke, M., see Dullin

Kapitaniak, T. \& Chua, L.O., Hyperchaotic attractors of unidirectionally-coupled Chua's circuits

Kapitaniak, T., Chua, L.O. \& Zhong, G.-Q., Experimental synchronization of chaos using continuous control

Karakatsanis, L,, see Pavlos

Katz, A.L. \& Dowell, E.H., From single well chaos to cross well chaos: A detailed explanation in terms of manifold intersections

Kawakami, H., see Mira

Kelso, J.A.S., see Ding

Kilias, T., Generation of pseudo-chaotic sequences $\quad 709$

Kim, Y.I., see Woo 137

Klingshirn, C., see Zhang

Knudsen, C., see Olesen

Kocarev, L. \& Tasev, Z., Analytical description of a fractal set generated by the time-delayed Chua's circuit

Kocarev, Lj., Dimovski, D., Tasev, Z. \& Chua, L.O., Knotted periodic orbits in Chua's circuit

Krinsky, V., see Biktashev

Krinsky, V.I., see Gómez-Gesteira

Krinsky, V.I., see Muñuzuri

Kurths, J., see Anishchenko

Kuznetsov, Yu A., see Champneys

L.'Heureux, I., see Nolte

Lakshmanan, M., see Murali

Latoussakis, J.B., see Pavlos

Lauterborn, W., see Englisch

Lauterborn, W., see Parlitz

Lee, E.-K., see Woo

Lesser, M., Nonlinear modeling of complex mechanical systems

Lichtenberg, A.J., see de Sousa Vieira

Lichtenberg, A.J., see de Sousa Vieira

Lieberman, M.A., see de Sousa Vieira

Lieberman, M.A., see de Sousa Vieira

Lisboa, J.A., see Braun

Maistrenko, V., Maistrenko, Y. \& Sushko, I., Noninvertible twodimensional maps arising in radiophysics

Maistrenko, Y., see Maistrenko

Mancini, H.L., see Mindlin

Mancini, H.L., see Maza-Ozcoidi

Mar, G. \& St. Denis, P., Chaos in cooperation: Continuous-valued prisoner's dilemmas in infinite-valued logic
751

761 
Markus, M. \& Stavridis, K., Observation of chemical turbulence in the Belousov-Zhabotinsky reaction

Martienssen, W., see Heng

Martienssen, W., see Doerner

Martienssen, W., see Hübinger

Martinez-Mardones, J., Tiemann, R., Zeller, W. \& Pérez-García, $\mathrm{C}$. , Amplitude equation in polymeric fluid convection

Massaguer, J.M., see Prat

May, R.M., see Nowak

Maza-Ozcoidi, D., Burguete, J. \& Mancini, H.L., Experimental results on defects in one-dimensional heating patterns

McLachlan, R.I., see Atela

Mees, A.I., see Paskota

Mercader, I., see Prat

Millan-Rodríguez, J., Influence of defects in the hexagon-roll transition in convective layers

Milton, J.G., see Chu

Mindlin, G.B., Ondarguhu, T., Mancini, H.L., Pérez García, C. \& Garcimartin, A., Comparison of data from Benard-Marangoni convection in a square container with a model based on symmetry arguments

Mira, C., Fournier-Prunaret, D., Gardini, L., Kawakami, H. \& Cathala, J.C., Basin bifurcations of two-dimensional noninvertible maps: Fractalization of basins

Mira, C., see Qriouet

Móndragon C., R.J. \& Richter, P.H., Weak dissipation in a hamiltonian map

Moreau, M., see Gaveau

Morozov, A.D., On the structure of resonance zones and chaos in nonlinear parametric systems

Moss, F., Pierson, D. \& O'Gorman, D., Stochastic resonance: Tutorial and update

Mossayebi, F., see Qammar

Müller, S.C., see Nagy-Ungvarai

Muñuzuri, A.P., see Gómez-Gesteira

Muñuzuri, A.P., Pérez-Muñuzuri, V., Gómez-Gesteira, M., Krinsky, V.I. \& Pérez-Villar, V., Mechanism of parametric resonance of vortices in excitable media

Murakami, H., see Itoh

Murakami, H., see Itoh

Murakami, H., see Itoh

Murali, K., Lakshmanan, M. \& Chua, L.O., Bifurcation and chaos in the simplest dissipative non-autonomous circuit

Muzy, J.F., Bacry, E. \& Arneodo, A., The multifractal formalism revisited with wavelets

Nagy-Ungvarai, Zs. \& Muller, S.C., Characterization of wave front instabilities in the Belousov-Zhabotinsky reaction: An overview

Neiman, A.B., see Anishchenko

Nekorkin, V.I., see Afraimovich

Nekorkin, V.I. \& Velarde, M.G., Solitary waves, soliton bound states and chaos in a dissipative Korteweg-de Vries equation

Nepomnyashchy, A., Large-scale distortions of square patterns

Neufeld, M. \& Friedrich, R., Pattern formation in rotating Benard convection

Nolte, K.-G. \& L.'Heureux, I., On the numerical detection of codimension-3 bifurcations of periodic solutions (with application to optical bistability

Norris, J.W., The nonlinear Mathieu equation

Nowak, M.A., Bonhoeffer, S. \& May, R.M., More spatial games

O'Gorman, D., see Moss

Ogorzalek, M., see Dedieu

Okniński, A., Three-dimensional rotations and discrete-time dynamical systems: Discrete symmetries in chaos-order transitions
1233

751

761

773

1347

1369

33

1353

865

457

1369

1357

237

343

411

921

1297

401

1383

843

1257

1193

1721
Okuda, H. \& Tsuda, I., A coupled chaotic system with different time scales: Possible implications of observations by dynamical systems

Olesen, M.W. \& Knudsen, C., Destruction of dominant Arnol'd tongues in forced oscillators

1121

Ondarguhu, T., see Mindlin

Otsuka, K., see Ishiyama

Otsuka, K. \& Chern, J.-L., Variation of Lyapunov exponents on a strange attractor for spiking laser oscillation

Otterstedt, R.D., Jaeger, N.I. \& Plath, P.J., Various wave phenomena during the oscillatory electrodissolution of cobalt

Overill, R.E., see Wells

Packard, N.H., see Breeden

Pande, M.B., Singh, R. \& Gupta, S.D., Chaos in a piecewiselinear system under frequency switching

Papaioannou, G., see Pavlos

Papanyan, V.O. \& Grigoryan, Yu I., Chaotization of ionization waves in a discharge plasma

Papiernik, E., see Sornette

Parlitz, U., Zöller, R., Holzfuss, J. \& Lauterborn, W., Reconstructing physical variables and parameters from dynamical systems

Paskota, M., Mees, A.I. \& Teo, K.L., Stabilizing higher periodic orbits

Patsis, P.A. \& Zachilas, L., Using color and rotation for visualizing four-dimensional Poincaré cross sections: With applications to the orbital behavior of a three-dimensional Hamiltonian system

Pavlos, G.P., Karakatsanis, L., Latoussakis, J.B., Dialetis, D. \& Papaioannou, G., Chaotic analysis of a time series composed of seismic events recorded in Japan

Pérez García, C., see Mindlin

Pérez-García, C., see Fernández-Vela

Pérez-García, C., see Martinez-Mardones

Pérez-García, V.M. \& Guerra, J.M., Weak turbulence in a high Fresnel number dye laser

Pérez-Muñuzuri, V., see Hanusse

Pérez-Muñuzuri, V., see Gómez-Gesteira

Pérez-Muñuzuri, V., see Muñuzuri

Pérez-Muñuzuri, V., Alonso, M., Chua, L.O. \& Pérez-Villar, V., Resonance patterns in one-dimensional arrays of coupled nonlinear excitable systems

Pérez-Villar, V., see Gómez-Gesteira

Pérez-Villar, V., see Muñuzuri

Pérez-Villar, V., see Pérez-Muñuzuri

Perraud, J.J., see De Kepper

Perrone, A.L., see Arecchi

Petrov, V., Crowley, M.F. \& Showalter, K., An adaptive control algorithm for tracking unstable periodic orbits

Pierson, D., see Moss

Pivka, L., Zheleznyak, A.L. \& Chua, L.O., Amol'd tongues, Devil's staircase, and self-similarity in the driven Chua's circuit

Plath, P.J., see Otterstedt

Plaza, F, see Coullet

Pomeau, Y., Defects and waves in a model of superfluid

Popkov, A.F., Yarema, I.P. \& Eleonsky, V.M., Nonuniform nonlinear oscillations of a domain wall in uniaxial ferromagnet

Prat, J., Mercader, I. \& Massaguer, J.M., Symmetric large-scale velocity fields in two-dimensional thermal convection

Premoli, A., see Chialina

Puccione, G.P., see Arecchi

Puente, C.E., Deterministic fractal geometry and probability

Qammar, H.K. \& Mossayebi, F., System identification and modelbased control of a chaotic system
1053

1265

1041

311

701

87 
Qriouet, M. \& Mira, C., Fractional harmonic synchronization in the Duffing-Rayleigh differential equation

Ramazza, P.L., see Arecchi

Record, R.J., see Gardini

Residori, S., see Arecchi

Richter, P.H., see Móndragon C

Richter, P.H., see Dullin

Riecke, H., see Herrero

Rodríguez-Lozano, A., see Del Río

Roytburd, V., see Frankel

Rudovics, B., see De Kepper

Rul'kov, N.F., see Del Río

Safonova, M.A., see Anishchenko

Safonova, M.A., see Anishchenko

Sagues, F., see Careta

Sakai, K. \& Aihara, K., Nonlinear vibrations in an agricultural implement system

Salán, J., see González-Viñas

Sancho, J.M., see Careta

Sancho, J.M., see García-Ojalvo

Sharkovsky, A.N., Ideal turbulence in an idealized time-delayed Chua's circuit

Shil'nikov, L.P., Chua's circuit: Rigorous results and future problems

Short, K.M., Steps towards unmasking secure communications

Showalter, K., see Petrov

Singh, R., see Pande

Soliman, M.S., Predicting regimes of indeterminate jumps to resonance by assessing fractal boundaries in control space

Sornette, D., Ferre, F. \& Papiernik, E., Mathematical model of human gestation and parturition: Implications for early diagnosis of prematurity and postmaturity

St. Denis, P., see Mar

Stämpfle, M., Controlling chaos through iteration sequences and interpolation techniques

Starmer, C.F., see Gómez-Gesteira

Stavridis, K., see Markus

Steffen, J., see Zhang

Sumida, T., Tahara, T. \& Iwanaga, H., Physiological significance of the Shil'nikov phenomenon in the focal accommodation system of human eyes

Sushko, I., see Maistrenko

Sweby, P.K, see Yee

Tahara, T, see Sumida

Tam, S.-W., see Ding

Tasev, Z., see Kocarev
Tasev, Z, see Kocarev

Teo, K.L., see Paskota

Tesi, A., de Angeli, A. \& Genesio, R., On system decomposition for synchronizing chaos

Tiemann, R., see Martinez-Mardones 1347

Tsuda, I., see Okuda

Uzer, T., see von Milczewski

Valsamma, K.M. \& Babu Joseph, K., Trajectory scaling function of polynomial circle maps

van Putten, M.H.P.M., A two-dimensional blast wave in relativistic magnetohydrodynamics

Velarde, M.G., see Del Río

Velarde, M.G., see Christov

Velarde, M.G., see Nekorkin

Volkovskii, A.R., see Del Río

Von Milczewski, J., Diercksen, G.H.F. \& Uzer, T., Classical dynamics of Rydberg electrons in crossed fields: The structure of phase space and chaos-order alternations

Wells, A.L.J. \& Overill, R.E., The extension of the FeigenbaumCvitanovic function to the complex plane

Werner, B., see Gatermann

Willeboordse, F.H., Encoding of traveling waves in a coupled map lattice

Woo, H.-J., Lee, E.-K. \& Kim, Y.I., Effect of perturbation on the area preservation map

Wu, C.W. \& Chua, L.O., A unified framework for synchronization and control of dynamical systems

1011

905

471

Yee, H.C. \& Sweby, P.K., Global asymptotic behavior of iterative implicit schemes

Zachilas, L., see Patsis

1579

1399

Zadorozhnii, V.I., see Dolinchuk

Zeller, W., see Martinez-Mardones

Zeng, W., see Glass

1525

1347

1061

1697

1193

1233

1375

Thang, F., Grohs, J., Steffen, J., Issler, H. \& Klingshirn, C., Selfoscillations and optical chaos of an induced absorber in coupled cavities

Zheleznyak, A.L. \& Chua, L.O., Coexistence of low- and highdimensional spatiotemporal chaos in a chain of dissipatively coupled Chua's circuits

Zheleznyak, A.L., see Pivka

Zhong, G.-Q., see Kapitaniak

Zimmerman, W.B., A weakly nonlinear instability of miscible displacements in a rectilinear porous media flow

Zöller, R., see Parlitz 